\title{
ELEMENTARILY EQUIVALENT FIELDS WITH INEQUIVALENT PERFECT CLOSURES
}

\author{
CARLOS R. VIDELA
}

\begin{abstract}
We give a counterexample to the following conjecture due to $\mathrm{L}$. V. den
\end{abstract} Dries: Let $F, L$ be two fields of characteristic $p$. If $F \equiv L$ then $F^{1 / p^{\infty}} \equiv L^{1 / p^{\infty}}$

Introduction. Van den Dries conjectures in [3, Stellingen 4] that elementarily equivalent fields have elementarily equivalent perfect closures. We will give a counterexample using a predicate introduced by Cherlin [1] in the study of definability in power series fields of nonzero characteristic.

Our counterexample is as follows: Fix a prime number $p$. Let $\tilde{\mathbf{F}}_{p}$ be the algebraic closure of the prime field $\mathbf{F}_{p}$, let $K_{0}=\tilde{\mathbf{F}}_{p}(t)$ be the rational function field, and let $L_{0}=K_{0}^{*}$ be a nonstandard extension of $K_{0}$, e.g. an ultrapower of $K_{0}$. In particular $K_{0} \equiv L_{0}$. We will prove:

THEOREM. The perfect closures of $K_{0}$ and $L_{0}$ are not elementarily equivalent.

The idea behind the proof is the following: we will relate the $p^{n}$ th powers of $t$, for $n$ an integer, to the $p^{n}$ th roots of $t$ in the perfect closure $K=K p^{-\infty}$ of $K_{0}$. However the $p^{N}$ th powers of $t$ with $N$ infinite will not correspond to roots of $t$ in $L=L p^{-\infty}$.

We will produce a sentence $\Phi$ whose meaning is approximately

$$
\text { "For all } n \text { if } t^{p^{n}} \text { exists then } t^{1 / p^{n}} \text { exists." }
$$

$\Phi$ will hold in $K$ and fail in $L$.

We make extensive use of the polynomial $\tau(x)=x^{p}-x$. We will need first order definitions of the following three predicates defined in $K$.

(1) $\operatorname{Con}(x)$ : “ $x \in \tilde{\mathbf{F}}_{p}$,"

(2) $\operatorname{Reg}(x): “ \neg \exists b \in \tilde{\mathbf{F}}_{p}-\{0\}, b x \in \tau[K]$,"

(3) $\operatorname{Link}(y, x)$ : “ $\exists n \in \mathbf{Z}, a \in \tilde{\mathbf{F}}_{p}, y=x^{p^{n}}+a \& \operatorname{Reg}(x)$."

For $\operatorname{Con}(x)$ we can do the following: choose $n>2$ and relatively prime to $p$. Then the curve $E$ defined by the equation $x^{n}+y^{n}=1$ is nonrational [2, p. 7]. This shows that the only solutions to the equation in $K_{0}$ must be constants. The same is true of $K$ : for if $x_{0}, y_{0} \in K$ are such that $x_{0}^{n}+y_{0}^{n}=1$, then by taking $p^{k}$ th powers for $k$ large enough, we get

$$
\left(x_{0}^{p^{k}}\right)^{n}+\left(y_{0}^{p^{k}}\right)^{n}=1
$$

Received by the editors September 16, 1985. This paper was presented at the meeting of the Mid-Atlantic Mathematical Logic Seminar, Institute for Advanced Study, Princeton, New Jersey, December 15, 1985.

1980 Mathematics Subject Classification (1985 Revision). Primary 03C60, 12L12.

Key words and phrases. Elementary equivalence, valuation, nonstandard model.

(C)1987 American Mathematical Society $0002-9939 / 87 \$ 1.00+\$ .25$ per page 
with $x p^{k}, y_{0}^{p^{k}} \in K_{0}$. Hence $x_{0}$ and $y_{0}$ are constants. We have shown that $\operatorname{Con}(x)$ defines the same set in $K_{0}$ and in $K$, namely $\tilde{\mathbf{F}}_{p}$. $\operatorname{Reg}(x)$, read " $x$ is regular," is visibly first order definable. We will use the following fact: Let $x \in K_{0}$. Then $K_{0}=\operatorname{Reg}(x) \Leftrightarrow K \vDash \operatorname{Reg}(x)$. A proof of this is implicit in Lemma 1 below.

The definition of $\operatorname{Link}(y, x)$ is as follows: First define a predicate $L_{0}(y, x)$ in the following way:

$$
\forall a \in \tilde{\mathbf{F}}_{p} \exists ! b \in \tilde{\mathbf{F}}_{p}(a y-b x \in \tau[K]) .
$$

Notice that $L_{0}(y, x)$ implies $x$ is regular: take $a=0$. When $L_{0}(y, x)$ holds, we write $y[a]$ for the element $b$ satisfying $a y-b x \in \tau[K]$, and we define $\operatorname{Link}(y, x)$ by a first order formalization of

$$
\text { “ } L_{0}(y, x) \& \forall a_{1}, a_{2} \in \tilde{\mathbf{F}}_{p}\left(y\left[a_{1} a_{2}\right]=y\left[a_{1}\right] y\left[a_{2}\right]\right) . "
$$

We will prove later that this has the intended meaning in $K$. Notice also that $\tau[K]$ is an additive abelian group and that, if $L_{0}(y, x)$, then the map $a \mapsto y[a]$ is an abelian group homomorphism (so that $\operatorname{Link}(y, x)$ says it is a field isomorphism too).

We can now write down the sentence $\Phi$ :

$$
\begin{aligned}
& \forall x, y([\operatorname{Link}(y, x) \& \neg \operatorname{Con}(y-x) \& \forall a(\operatorname{Con}(a) \Rightarrow \operatorname{Reg}(x(y-a)))] \\
& \quad \Rightarrow \exists z, b[\operatorname{Con}(b) \& \operatorname{Link}(z, x) \& \operatorname{Link}(x z, x(y-b)) \& \neg \operatorname{Con}(y-z)]) .
\end{aligned}
$$

We will show that $\Phi$ is true in $K$; on the other hand taking $x=t$ we will see that $\Phi$ is false in $L$. It turns out that the content of $\Phi$ is approximately

$$
\forall x \forall n \neq 0 \forall y \in x^{p^{n}}+\tilde{\mathbf{F}}_{p}\left(\exists z, a \in \tilde{\mathbf{F}}_{p}\left((x z)^{p^{n}}=x(y-a) \& x-z^{p^{n}} \in \tilde{\mathbf{F}}_{p}\right)\right) .
$$

\section{The interpretation of $\Phi$ in $K$.}

Lemma 1. For $x, y \in K_{0}$, if $K \vDash \operatorname{Link}(y, x)$, then $K_{0} \vDash \operatorname{Link}(y, x)$.

Proof. Let $a \in \tilde{\mathbf{F}}_{p}$. Then there exist $b \in \tilde{\mathbf{F}}_{p}$ and $w \in K$ such that $a y-b x=w^{p}$ $-w$. Note that $w$ is inseparable over $K_{0}$. Since $w^{p}-w \in K_{0}$, it follows that $K_{0}(w)=K_{0}\left(w^{p}\right)=K_{0}\left(w^{p^{2}}\right)=\cdots$. Hence $w$ is separable over $K_{0}$ and so must be in $K_{0}$.

We will show that $\operatorname{Link}(y, x)$ has the intended meaning by proving that the map $a \mapsto y[a]$ is a first-order definable automorphism of $\tilde{\mathbf{F}}_{p}$ with parameters in $\mathbf{F}_{p}$. It will then follow that $y[a]=a^{p^{n}}$ for some $n \in \mathbf{Z}$. Finally, we show that this implies $y-x^{p^{-n}}$ is a constant.

For $x \in K_{0}$ let $P_{x}$ be the set of all poles (finite and at infinity) $\not<$ of $x$. For each $\not$ let $\tilde{\mathbf{F}}_{p}\left(\left(t_{p}\right)\right)$ be the completion of $K_{0}$ with respect to the valuation $V_{k}$ determined by p. $K_{0}$ is embedded in $\tilde{\mathbf{F}}_{p}\left(\left(t_{p}\right)\right)$. We will make use of the following fact [1, p. 103]: Let $x \in \tilde{\mathbf{F}}_{p}\left(\left(t_{p}\right)\right), x=\sum x_{i} t_{p}^{i}$, then $x \in \tau\left[\tilde{\mathbf{F}}_{p}\left(\left(t_{p}\right)\right)\right]$ if and only if the following conditions $\left(c_{i}\right)$ for $i<0, i \neq 0(\bmod p)$ are satisfied:

$$
\sum_{n \geqslant 0}\left(x_{i p} n\right)^{1 / p^{n}}=0
$$

REMARK. Notice that if $\nu_{p}(x) \geqslant 0$, then $x \in \tau\left[\tilde{\mathbf{F}}_{p}\left(\left(t_{p}\right)\right)\right]$, since the left-hand side of $\left(c_{i}\right)$ is zero. 
Lemma 2. Suppose $x, y \in K_{0}$ and $\operatorname{Link}(y, x)$. Then the map $a \rightarrow y[a]$ is a first order definable (with parameters) automorphism of $\tilde{\mathbf{F}}_{p}$.

Proof. Fix $\not p \in P_{x}$ and let $a \in \tilde{\mathbf{F}}_{p}$. There exists $b \in \tilde{\mathbf{F}}_{p}$ such that $a y-b x \in \tau\left[K_{0}\right]$. In the completion $\tilde{\mathbf{F}}_{p}\left(\left(t_{p}\right)\right)$, the finitely many equations $\left(c_{i}\right)$ define a first-order formula $\theta_{p}\left(u, w, \bar{c}_{p}\right)$, where $\bar{c}_{p}$ is a sequence of parameters (essentially the coefficients in the principal parts of the Laurent expansions of $y$ and $x$ ), such that, for $a$ and $b$ as above $\theta_{p}\left(a, b, \bar{c}_{p}\right)$ holds.

Define $\psi(u, w)=\Lambda_{p \in P_{x}} \theta_{p}\left(u, w, \bar{c}_{p}\right)$. We claim that for any $a \in \tilde{\mathbf{F}}_{p}, b=y[a] \Leftrightarrow$ $\psi(a, b)$. The claim follows if we show that given $a \in \tilde{\mathbf{F}}_{p}$ there exist one and only one $b \in \tilde{\mathbf{F}}_{p}$ with $\psi(a, b)$. So suppose $\psi(a, b)$ and $\psi\left(a, b^{\prime}\right)$. This implies that $\left(b-b^{\prime}\right) x \in$ $\tau\left[\tilde{\mathbf{F}}_{p}\left(\left(t_{h}\right)\right)\right]$ for all $\not \in P_{x}$, hence $\left(b-b^{\prime}\right) x \in \tau\left[K_{0}\right]$ and since $x$ is regular, $b=b^{\prime}$. For the existence, take $b=y[a]$.

LEMMA 3. Let $F$ be an algebraically closed field of characteristic $p$. Then

(i) if $\phi: F \rightarrow F$ is an automorphism of $F$ definable over $F$, there exists $r(x) \in F(x)$ and $n \in \mathbf{N}$ such that, except for finitely many elements of $F$, we have $\phi(a)=r(a)^{1 / p^{n}}$.

(ii) if $\phi: F \rightarrow F$ is an automorphism of $F$ and $r(x) \in F(x)$ is such that $r(a)=\phi(a)$ for all $a \in F$ except for a finite set, then $r(x)=x^{p^{k}}$ for some $k \in \mathbf{Z}$.

Proof. Let $t$ be transcendental over $F$ and let $\overline{F(t)}$ be the algebraic closure of

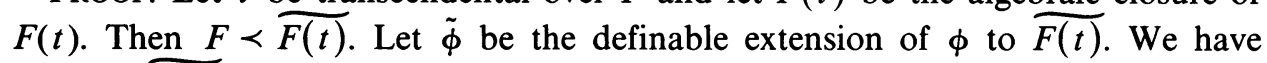
$\tilde{\phi} \in$ Aut $\overline{F(t)}$.

Let $G=\operatorname{Gal}(\overline{F(t)} / F(t)), \psi(x, y, \bar{c})$ be a definition of $\phi$ over $F$. Then for any $\sigma \in G$ we have

$$
\begin{aligned}
\widetilde{F(t)} & \vDash \psi(t, \tilde{\phi}(t), \bar{c}) \\
& \Rightarrow \widetilde{F(t)} \vDash \psi(\sigma(t), \sigma(\tilde{\phi}(t)), \sigma(\bar{c})) \\
& \Rightarrow \widetilde{F(t)} \vDash \psi(t, \sigma(\tilde{\phi}(t)), \bar{c}) .
\end{aligned}
$$

Hence $\sigma(\tilde{\phi}(t))=\tilde{\phi}(t)$, i.e. $\tilde{\phi}(t) \in F(t)^{1 / p^{\infty}}$. This proves $\tilde{\phi}(t)=r(t)^{1 / p^{n}}$ for some $r(x) \in F(x)$ and $n \in \mathbf{N}$. Next we show that the set $A:=\left\{a \in F \mid \tilde{\phi}(a)=r(a)^{1 / p^{n}}\right\}$ has finite complement. This follows from the following two facts:

(a) $A$ is infinite,

(b) $F$ is strongly minimal.

To prove (a) note that for any finite set $S \subset F$

$$
\widetilde{F(t)} \vDash \exists x\left(\tilde{\phi}(x)=r(x)^{1 / p^{n}} \& x \notin S\right),
$$

hence $F \vDash \exists x\left(\tilde{\phi}(x)=r(x)^{1 / p^{n}} \& x \notin S\right)$.

To prove (ii) note that for $t$ transcendental (as above) we have infinitely many $a \in F$ such that

$$
r(a t)=r(a) r(t) \text {. }
$$

Hence the set of zeros $Z$ and the set of poles $P$ of $r(x)$ are closed under multiplication by $a$ for infinitely many $a \in F$. Since $Z$ and $P$ are finite sets we have $Z=P=\{0\} \cup\{\infty\}$, and so $r(x)=x^{p^{n}}$. 
It follows that $\phi(x)=r(x)$ for all $x \in F$.

COROllary. With the assumptions of Lemma 3 there exists $k \in \mathbf{Z}$ such that $y[a]=a^{p^{k}}$.

The following fact will be used in the proof of Proposition 1.

FACT. If $y, x \in K_{0}$ and $\operatorname{Link}(y, x)$, then $P_{x}=P_{y}$.

ProOF. By symmetry it is enough to show that $P_{y} \subseteq P_{x}$. Suppose not. Let $\not \in \in P_{y}-P_{x}$. Then, since $\nu_{p}(x) \geqslant 0$, for all $a \in \tilde{\mathbf{F}}_{p}$ we have ay $\in \tau\left[\tilde{\mathbf{F}}_{p}\left(\left(t_{p}\right)\right)\right]$. Since $\tilde{\mathbf{F}}_{p}$ is infinite, it follows by using equation $\left(c_{i}\right)$ that the principal part of the Laurent expansion of $y$ must be zero, hence $\nu_{\mu}(y) \geqslant 0$, a contradiction.

Proposition 1. Let $x, y \in K_{0}$ and assume that $x$ is regular. Then

$$
K_{0} \vDash \operatorname{Link}(y, x) \Leftrightarrow \exists n \in \mathbf{Z} \text { and } c \in \tilde{\mathbf{F}}_{p} \text { such that } y=x^{p^{n}}+c .
$$

Proof. Assume $L(y, x)$. The corollary to Lemma 4 implies that $y[a]=b=a^{p^{n}}$ for some $n \in \mathbf{Z}$. Hence $a y-a^{p^{n}} x \in \tau\left[\tilde{\mathbf{F}}_{p}\left(\left(t_{\not}\right)\right)\right]$ for all $\not \in \in P_{x}$ and all $a \in \tilde{\mathbf{F}}_{p}$. We claim that $\nu_{p}\left(y-x^{p^{-n}}\right) \geqslant 0$ for all $p \in P_{x}$. Conditions $\left(c_{i}\right)$ yield the following

$$
\sum_{k=0}^{N_{0}}\left(a y_{i p^{k}}-a^{p^{n}} x_{i p^{k}}\right)^{1 / p^{k}}=0
$$

where $N_{0} \in \mathbf{N}$ and the $y_{i p^{k}}, x_{i p^{k}}$ are the coefficients appearing in the principal part of the Laurent expansions of $y$ and $x$. Taking $p^{N_{0}}$ th powers in the above equation gives us a polynomial in $a$, which is identically zero. By distinguishing the possibilities $N_{0}>n, N_{0}=n$, and $N_{0}<n$ one shows that $y_{i}^{p^{n}}=x_{i p^{n}}$ for all $i<0$.

To conclude the proof note that a pole of $y-x^{p^{-n}}$ is a pole of $y$ or $x$ and by the fact stated before the proposition it is a pole of $x$. Hence $y-x^{p^{-n}}$ has no poles at all and so must be a constant. For the other implication take $b=a^{p^{-n}}$; uniqueness is a consequence of the regularity of $x$.

COROllary. For $y, x \in K, x$ regular,

$$
K \vDash \operatorname{Link}(y, x) \Leftrightarrow \exists n \in \mathbf{Z} \text { and } a \in \tilde{\mathbf{F}}_{p} \text { such that } y=x^{p^{n}}+a .
$$

Proof. Taking $p^{n}$ th powers yields an automorphism of $K$. Therefore we may take $y, x \in K_{0}$ and note that regularity over $K$ or $K_{0}$ is the same. The result follows from Lemma 1 and Proposition 1.

Proposition 2. $K \vDash \Phi$.

Proof. Let $x, y \in K$ satisfy $\operatorname{Link}(y, x)$. Then $y=x^{p^{n}}+a$ for some $n$ and $a$. We may assume $n \neq 0$ since otherwise $y-x$ is a constant. Take $b=a$ and $z=x^{p^{-n}}$. Clearly $\operatorname{Link}(z, x)$. Then $x(y-b)=x x^{p^{n}}$ and $x z=x x^{p^{-n}}=\left(x x^{p^{n}}\right)^{p^{-n}}$. We have that $y-z$ is not constant and $\operatorname{Link}(x z, x(y-b))$.

2. The interpretation of $\Phi$ in $L$. The set defined by $\operatorname{Con}(x)$ in $L$ and in $L_{0}$ is the same; denote it by $\tilde{\mathbf{F}}_{p}^{*}$. Lemma 1 remains true for the pair $L_{0}, L$. By transfer applied to the structure $\left(L_{0}, \mathbf{Z}^{*}, \tilde{\mathbf{F}}_{p}^{*}\right)$ we get that, for $x, y \in L, x$ regular,

$$
L \vDash \operatorname{Link}(y, x) \Leftrightarrow \exists n \in \mathbf{Z}^{*}, a \in \tilde{\mathbf{F}}_{p}^{*} \text { such that } y=x^{p^{n}}+a .
$$


Proposition 3. $L \not \neq \Phi$.

Proof. Take $x=t$ and $y=t^{p^{N}}$ with $N$ infinite and positive. Clearly $x$ is regular and linked to $y$. Next, for any $a \in \tilde{\mathbf{F}}_{p}^{*}$, we claim that $t\left(t^{p^{N}}-a\right)$ is regular. Otherwise, by transfer we could have $b t\left(t^{p^{n}}-a^{\prime}\right) \in \tau\left[K_{0}\right]$ with $b \neq 0, b, a^{\prime} \in \tilde{\mathbf{F}}_{p}$, and $n$ a positive integer. But this is impossible. So, assume there exists $z, a \in \tilde{\mathbf{F}}_{p}^{*}$ such that $\operatorname{Link}(z, t)$ and $\operatorname{Link}\left(t z, t\left(t^{p^{N}}-a\right)\right)$. Then $z=t^{p^{n}}+a^{\prime}, a^{\prime} \in \tilde{\mathbf{F}}_{p}^{*}, n \in \mathbf{Z}^{*}$. Notice that if $n<0$, then $n$ cannot be infinite since $L=\bigcup_{n \in w} L_{0}^{1 / p^{n}}$.

From $\operatorname{Link}\left(t z, t\left(t^{p^{N}}-a\right)\right)$ we get an equation of the form

$$
t\left(t^{p^{n}}+a^{\prime}\right)=\left(t\left(t^{p^{N}}-a\right)\right)^{p^{k}}+a^{\prime \prime}
$$

for some $a^{\prime \prime} \in \tilde{\mathbf{F}}_{p}^{*}$ and $k \in \mathbf{Z}^{*}$. Note that $a^{\prime \prime}$ must be equal to zero. We show (1) cannot hold.

Case 1. $a^{\prime}=0$, (or $a=0$ ). Then $a=0$ and we have $t^{p^{n}+1}=t^{p^{k}} t^{p^{N+k}}$, so $p^{n}+1=$ $p^{k}+p^{N+k}$. The equation has the following solutions: (a) $k=0, n=N$, and (b) $N+k=0, n=k$. (a) implies $y=z$ which we have excluded and (b) implies $n=-N$ which is also impossible as $N$ is infinite.

Case 2. $a^{\prime} \neq 0$ and $a \neq 0$. In this case $k=0$ so $N=n$ and $y-z=a$ which is impossible. Thus $\Phi$ is false in $L$, and $K \not \equiv L$.

I thank G. Cherlin for his help in the preparation of this paper.

\section{REFERENCES}

1. G. Cherlin, Definability in power series rings of nonzero characteristic models and sets, Lecture Notes in Math., vol. 1103, Springer-Verlag, Berlin and New York, 1984.

2. I. R. Shafarevich, Basic algebraic geometry, Springer-Verlag, New York, 1977.

3. L. Van den Dries, Model theory of fields, Thesis, Utrecht 1978, Stellingen 4.

Department of Mathematics, Rutgers University, New Brunswick, New Jersey 08903 